\title{
Candida parapsilosis, a rare cause of fungal melanonychia
}

\author{
Min Kyun An, M.D., Kwang Ho Kim, M.D., Ph.D. \\ Department of Dermatology, College of Medicine, Hallym University, Anyang, Korea
}

\section{Introduetion}

Melanonychia means tan, brown, or black pigmentation within the nail plate. Fungal melanoncyhia is rare but fungal infection may be associated with longitudinal melanonychia caused by melanocytic lesions. Based on the increasing prevalence of Candida parapsilosis among onychomycosis patients, we conducted the study to assess the characteristics of fungal melanonychia caused by Candida parapsilosis.

\section{Case presentition}

55-year old female patient was refered for melanonychia on the both great toenails (Figure 1). For the diagnosis of fungal melanonychia, direct microscopic examination of scrapings from the affected nails in $15 \%$ potassium hydroxide $(\mathrm{KOH})$ and cultures at $25^{\circ} \mathrm{C}$ on Sabouraud's dextrose agar containing chloramphenicol with and with out cycloheximide were performed. And dermoscopic examination(Figure 2), and gene sequencing, extraction-gDNA and PCR amplification were conducted (Macrogen Inc., Korea). KOH was positive and Candida parapsilosis was isloated in both fungus culture (Figure 3) and gDNA sequencing. After terbinafine daily 250mg treatment, remained lesion on the right great toe nail still showed positive result of $\mathrm{KOH}$. Candida parapsilosis was isolated again in both fungus culture and gDNA sequencing. $3^{\text {rd }}$ cycle of itraconazole was finished, her clinical features have improved (Figure 4).

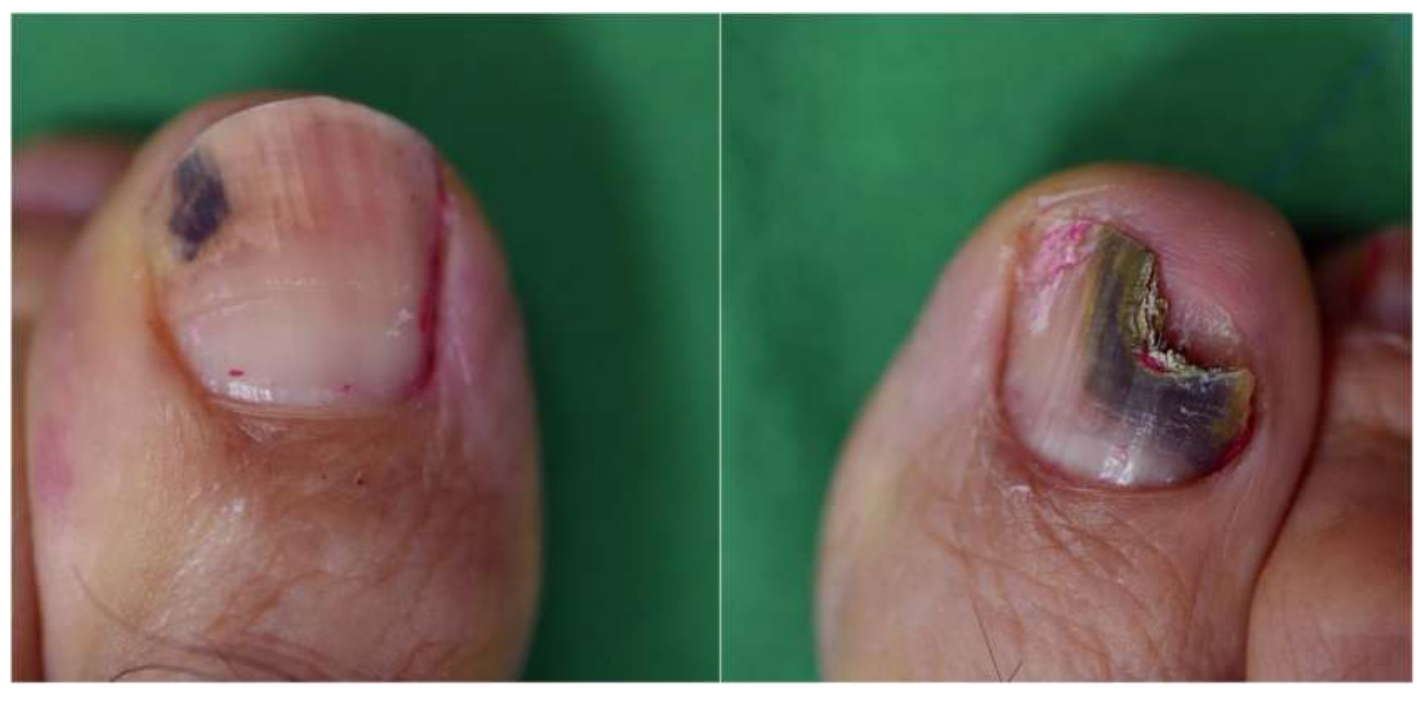

Fig 1. Melanonychia on the both great toenails.

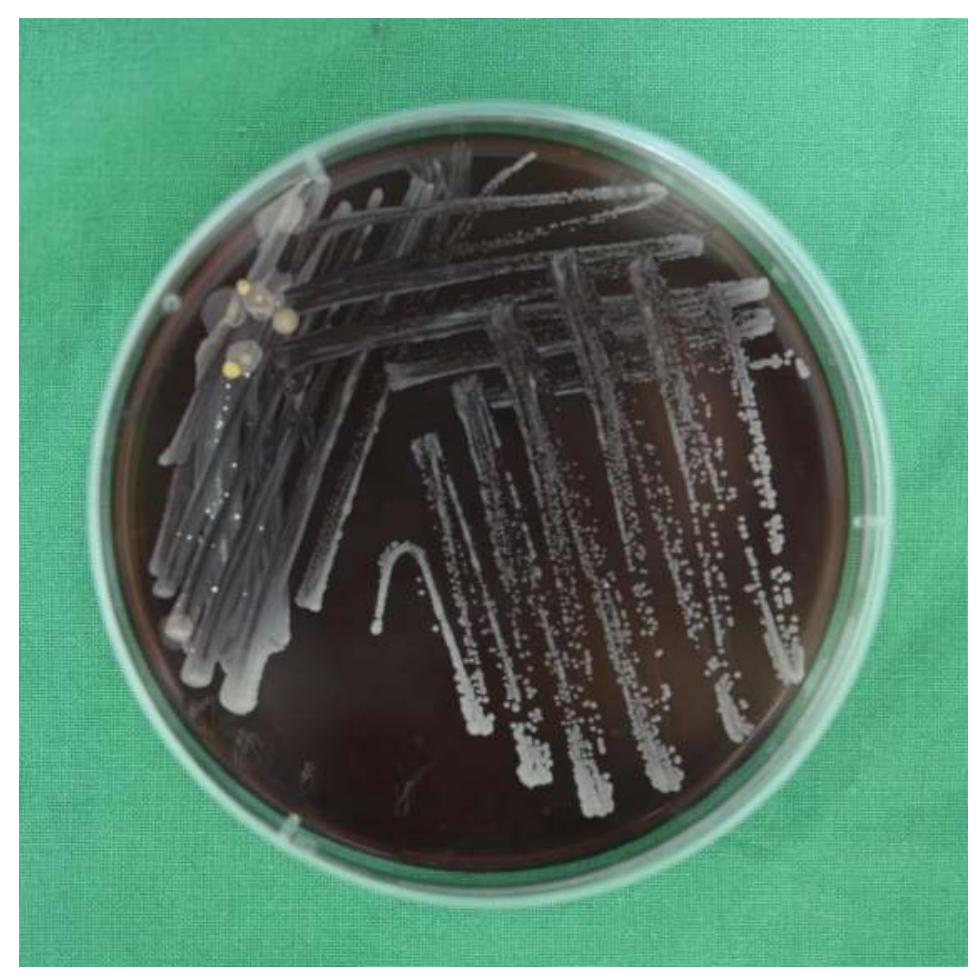

Fig 3. Candida parapsilosis was isloated in both fungus culture.

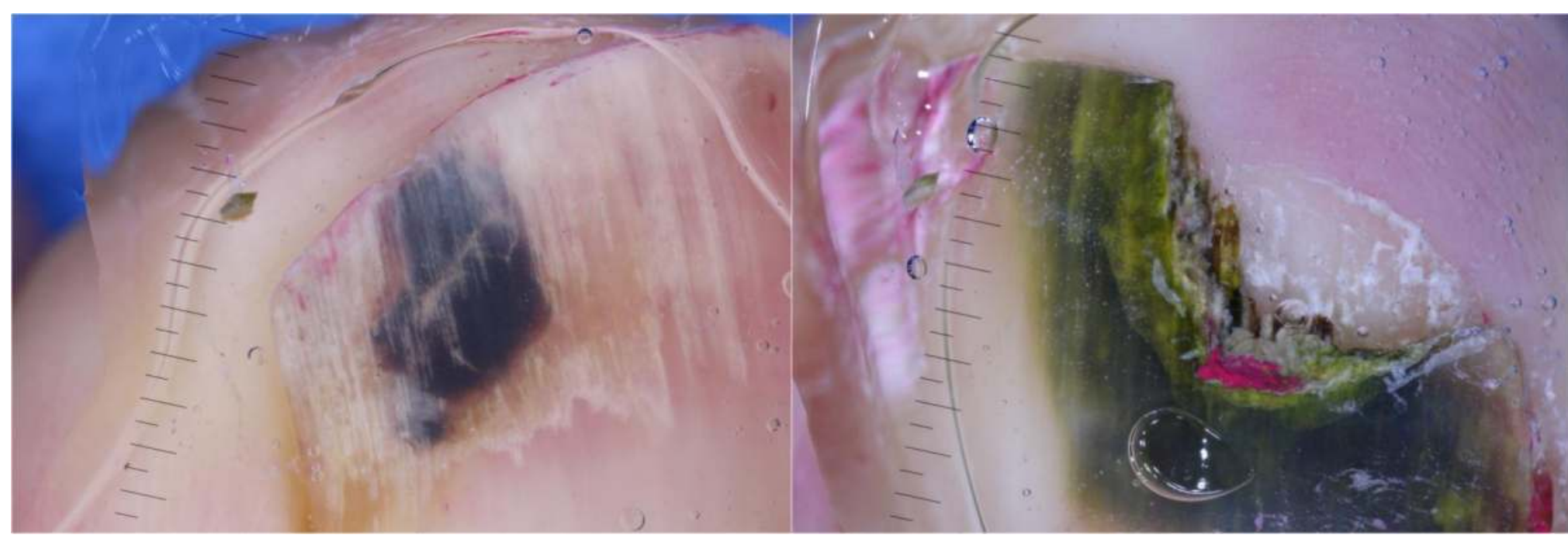

Fig 2. Dermoscopy images showing multicolored pigmentation, longitudinal pigmentation.

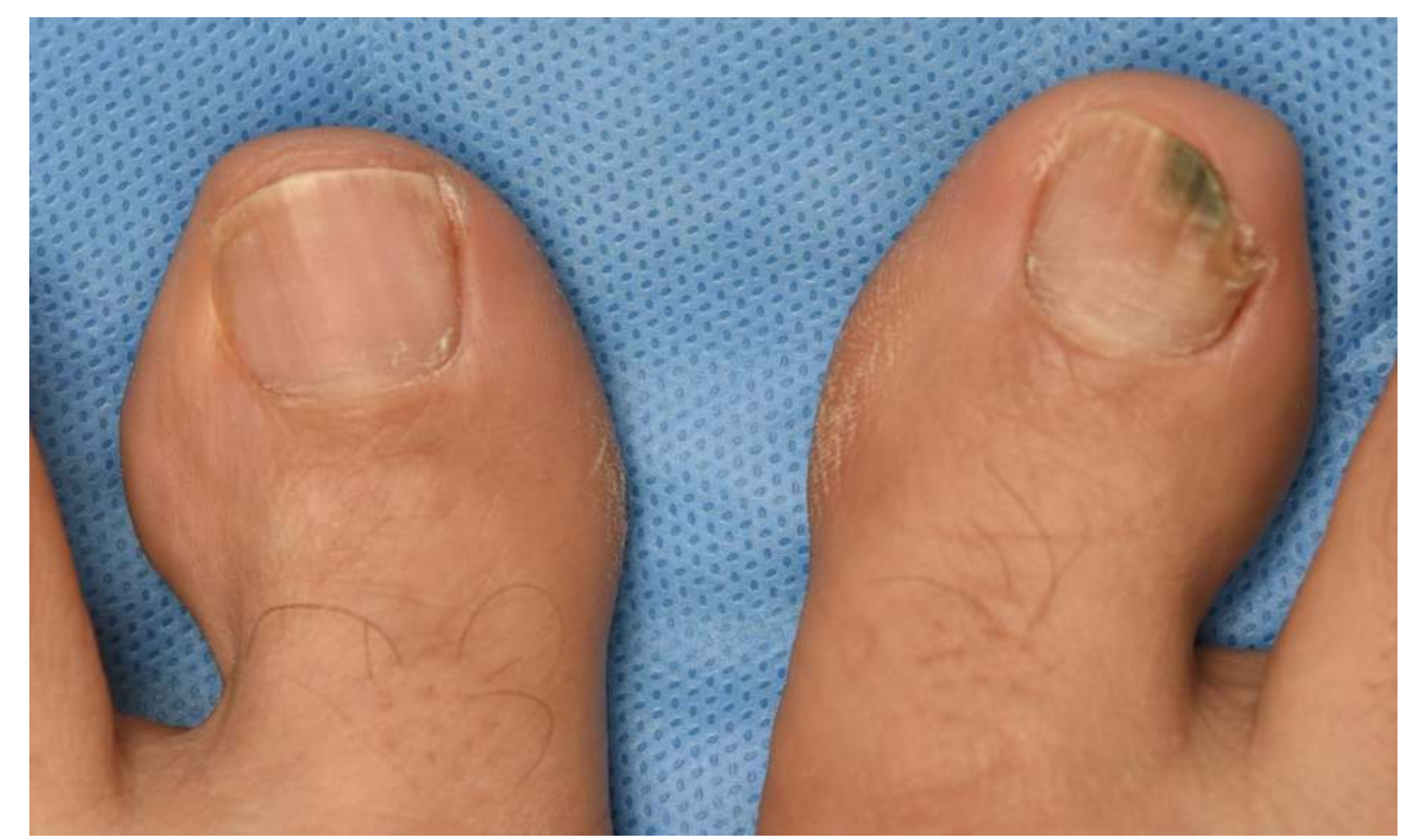

Fig 4. Clinical features improved after $3^{\text {rd }}$ cycle of itraconazole.

\section{Disenssion}

Fungal melanonychia is a rare but cannot be overlooked cause of melanonychia. A pigmented and dystrophic nail should raise the suspicion of melanoma, and this should be excluded before considering other diagnoses. In cases with features such as melanonychia on the distal portion of the nail and subungual hyperkeratosis, fungal melanonychia is also relatively easy to diagnosis. However, as reported in the literature, if the pigmentation involves the proximal portion of the nail and there is no Hutchinson sign, differentiating between melanoma and fungal melanonychia may be difficult. The main dermoscopic pattern of fungal melanonychia is homogeneous pigmentation. And matt black, white, yellow to brown pigmented aggregates, black reverse triangle (wider at the distal than the proximal end), superficial transverse striation and blurred appearance were the other features.

Fungal melanonychia caused by Candida is very rare and only a few cases have been reported in the literature. The mechanism caused by Candida is not known, and it is hypothesized that paronychia-induced melanocyte stimulation induces pigmentation. Recent literature reports indicate that Candida albicans synthesizes melanin. However, there is no study of what causes in vivo the melanin synthesis of Candida. A unique feature of fungal melanosis caused by Candida parapsilosis reported in the literature is that the patient has traumatic dystrophy of the nail and gardening activity. Because Candida parapsilosis is a fungus isolated from soil, gardening is a risk factor for infection. On the other hand, there was no specific history in this case and there was no risk factor for Candida parapsilosis. In this case, irregular matt white, yellow or brown pigmentation seen as pigmented areas, longitudinal pigmentation.

Until now, there is no definite treatment option established for Candida parapsilosis onychomycosis. Further study for appropriate antifungal agent should be conducted, considering increasing prevalence of Candida parapsilosis onychomycosis patients. 